\title{
Resolved Stellar Populations of the interacting galaxies of the M81 group
}

\section{Sakurako Okamoto, Nobuo Arimoto, Annette M.N. Ferguson, Edouard J. Bernard, Mike J. Irwin, Yoshihiko Yamada and Yousuke Utsumi}

Shanghai Astronomical Observatory, Chinese Academy of Sciences,

80, Nandan Rd. Shanghai, China

email: sakurako.okamoto@gmail.com

\begin{abstract}
We present the results from the state-of-the-art wide-field survey of the M81 galaxy group that we are conducting with Hyper Suprime-Cam on Subaru Telescope. Our photometry reaches about $2 \mathrm{mag}$ below the tip of the red giant branch (RGB) and reveals the spatial distribution of both old and young stars over an area of $5 \mathrm{deg}^{2}$ around the M81. The young main-sequence (MS) stars closely follow the HI distribution and can be found in a stellar stream between M81 and NGC 3077 and in numerous outlying stellar associations. Our survey also reveals for the first time the very extended $\left(>2 \times \mathrm{R}_{25}\right)$ halos of RGB stars around M81, M82, and NGC 3077, as well as faint tidal streams that link these systems. The gravitational interactions between M81, M82 and NGC 3077 galaxies induced star formation in tidally stripped gas, and also significantly perturbed the older stellar components leading to disturbed halo morphologies.
\end{abstract}

Keywords. galaxies: individual (M81, M82, NGC 3077), galaxies: interactions, galaxies: photometry, galaxies: stellar content, galaxies: structure

\section{Introduction}

M81, the large spiral galaxy located at 3.6 Mpc from the Milky Way is a prime target for wide-field mapping of its resolved stellar content beyond the Local Group. Spectacular neutral hydrogen images have demonstrated the significant tidal interactions between M81 and its two brightest neighbors, M82 and NGC 3077, which modelling suggests have taken place in the last 300 Myrs (e.g. Yun et al. 1994, Yun 1999). Deep photometry from the Hubble Space Telescope (HST) has been used to argue that the outlying HI concentrations Arp's Loop (AL), and Holmberg IX (HoIX) may be tidal dwarf galaxies formed as a result of these interactions (e.g. Makarova et al. 2002).

Wide-field images of $8 \mathrm{~m}$ telescope and deep HST pointings also reveal that the evidence for a faint, extended old stellar component beyond the bright optical disk of M81 (Barker et al. 2009, Monachesi et al. 2013). Chiboucas et al. (2013) confirmed 12 new dwarf satellites as members of the M81 group, discovered from a 65 deg $^{2}$ survey with CFHT/MegaCam. However, the global properties of the stellar populations throughout the M81 group are still poorly known since it requires both wide-field coverage and image sensitivity of instruments.

\section{Observation and Color Magnitude-Diagram}

We observed the region around M81 in the $g$ - and $i$-bands using 4 pointings of the new prime-focus imager, Hyper Suprime-Cam (HSC), on the Subaru Telescope (PI: S. Okamoto; Proposal ID: S14B-101). The observations were obtained as part of a survey to map the central region of M81 group with seven HSC pointings as shown in the 

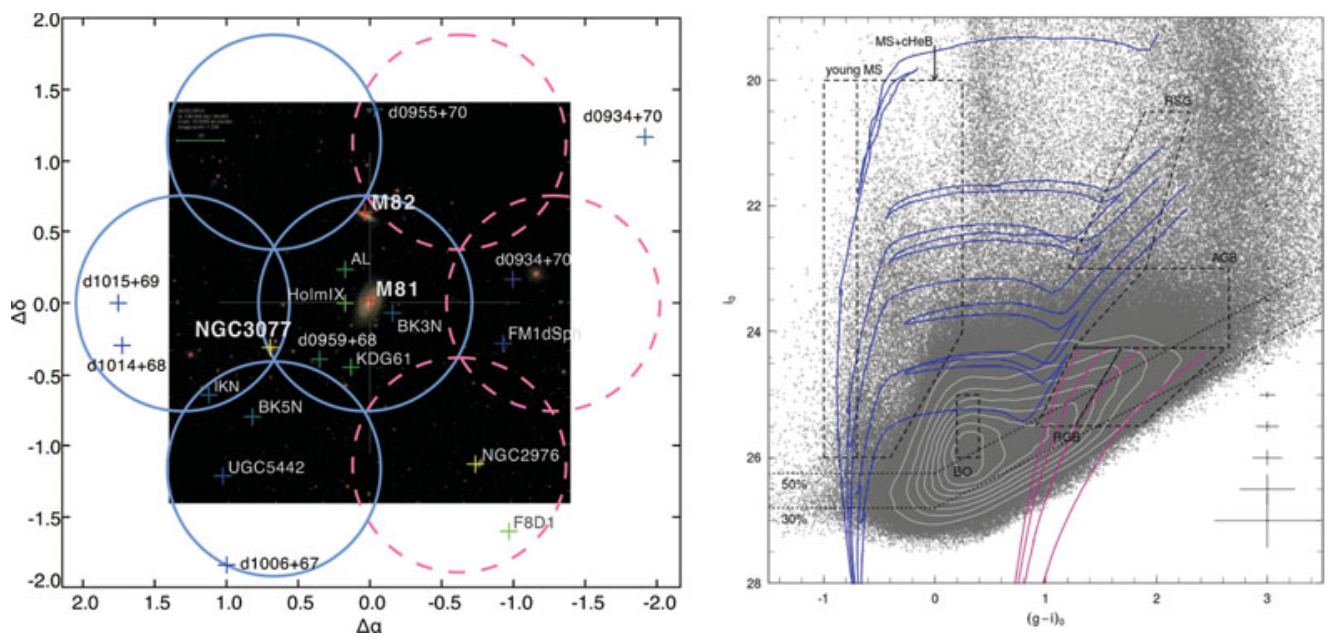

Figure 1. Left: The observed and planned HSC pointings around the M81 galaxy are shown as the blue solid circles and the magenta dashed circles, respectively, on the colour images taken from SDSS. The known member galaxies of M81 Group are marked as cross symbols. Right: Dereddened CMD of stellar objects located within the observed $5 \operatorname{deg}^{2}$ area. Theoretical isochrones are shown for a $12 \mathrm{Gyr}$ old population with $[\mathrm{M} / \mathrm{H}]=-2.2,-1.75,-1.3,-0.75$ from the left to the right (magenta) and for an $[\mathrm{M} / \mathrm{H}]=-0.75$ population with ages of $10,18,32,50,100,160$ Myr from top to bottom (blue).

left panel of Figure 1. The right panel of Figure 1 shows the resulting color-magnitude diagram (CMD) of roughly 930,000 de-reddend point sources found in the $5 \mathrm{deg}^{2}$ area area around M81. The detail of data reduction is described in Okamoto et al. (2015). Theoretical Padova isochrones are overlaid to aid in understanding the range of stellar populations (Bressan et al. 2012). The dashed boxes delineate the selection criteria for different stellar populations and are used to construct the maps of young and old stellar contents presented in Figure 2.

\section{Results and Discussion}

The left panel of Figure 2 shows the spatial distribution of stars in young MS and $\mathrm{MS}+\mathrm{cHeB}$ boxes of the right panel of Figure 1, which are color coded according to iband magnitude in a transparent manner so that colors of overlapping points represent the average color. Overall distribution of young stars agrees extremely well with those of the HI blobs, except for the stream at the northwest of NGC 3077 where few stars are seen. Bright stars are mainly located in the inner disk of M81, while most of young stars in the northwest side of M81, AL, HoIX, BK3N, the Garland, the stream between M81 and NGC 3077, and other debris features are fainter than $i_{0} \sim 24$ and have similar luminosity distributions each other, suggesting that star formation in these tidal features was synchronized and may have stopped about $30 \mathrm{Myr}$ ago.

The right panel of Figure 2 shows the contour map of red RGB stars. A tidal stream between M81 and M82 can clearly be seen, and the outer regions of M82 and NGC3077 exhibit an S-shaped morphology. The NGC 3077 halo is extended far beyond the R25 $\left(\mathrm{r}=2.7^{\prime}\right)$. The component in the northwest appears to reach a maximum projected radius from NGC 3077 of $\sim 65 \mathrm{kpc}$, but does not appear to trace the HI distribution in this region. Numerical modeling suggests the encounters between NGC 3077, M81, and M82 took place 200-300 Myr ago (Yun et al. 1999), which may not leave enough time 


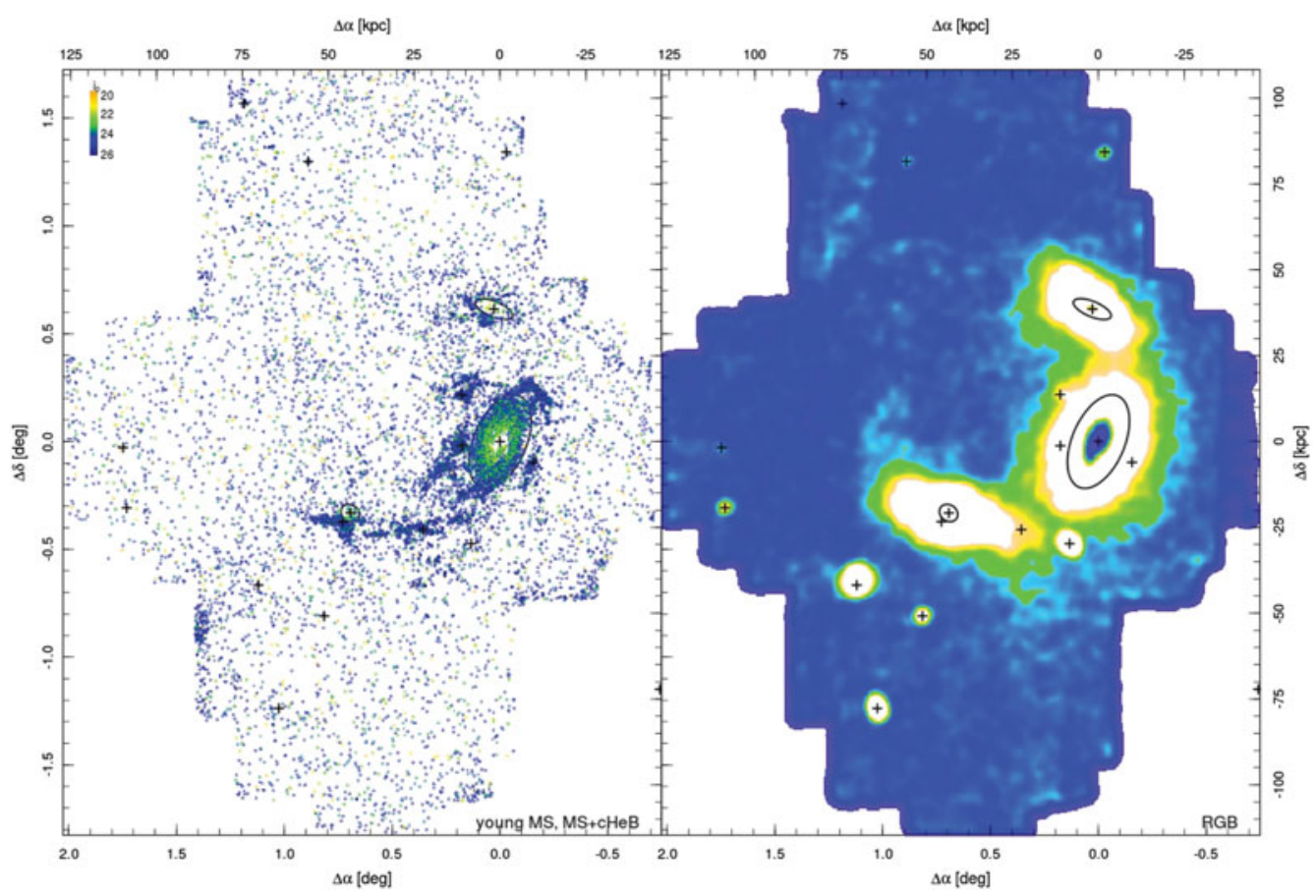

Figure 2. Left: the spatial distribution of MS and cHeB stars that are color coded according to the luminosity with transparency. Right: Iso-density contour map of red RGB stars, featured to faint structures up to $20 \sigma$ above the background level. The kernel density is estimated with the bandwidth of $1.2^{\prime}$. The solid lines are $\mathrm{R} 25$ radii of galaxies.

to restore equilibrium in the NGC 3077 halo. The dwarf galaxies such as IKN and BK5N cannot be seen in the maps of young stars, but appear as overdensities of old populations, implying they have not formed as a result of the recent interaction.

When we complete all seven paintings in the forthcoming HSC observation, the data will allow us to determine the true extent and nature of the intra-group debris and map the halos of the M81 group galaxies to unprecedented distances.

\section{References}

Barker, M. K., Ferguson, A. M. N., Irwin, M., Arimoto, N., \& Jablonka, P. 2009, AJ, 138, 1469

Bressan, A., Marigo, P., Girardi, L., Salasnich, B., Dal Cero, C., Rubele, S., \& Nanni, A. 2012, MNRAS, 427, 127

Bullock, J. S. \& Johnston, K. V. 2005, ApJ, 635, 931

Chiboucas, K., Jacobs, B. A., Tully, R. B., \& Karachentsev, I. D. 2013, AJ, 146, 126

Fitzpatrick, E. L. 1999, PASP, 111, 63

Makarova, L. N., Grebel, E. K., Karachentsev, I. D., Dolphin, A. E., Karachentseva, V. E., Sharina, M. E., Geisler, D., Guhathakurta, P., Hodge, P., Sarajedini, A., \& Seitzer, P. 2002, A\&A, 396, 473

Monachesi, A., Bell, E. F., Radburn-Smith, D. J., Vlajić, M., de Jong, R. S., Bailin.J., Dalcanton, J. J., Holwerda, B. W., \& Streich, D. 2013, ApJ, 766, 106

Okamoto, S., Arimoto, N., Ferguson, A. M. N., Bernard, E. J., Irwin, M. J., \& Yamada, Y. Utsumi, Y. 2015, ApJ(Letter), 809, L1

Schlafly, E. F. \& Finkbeiner, D. P. 2011, ApJ, 737, 103

Yun, M. S. 1999, in: J. E. Barnes \& D. B. Sanders (eds.), Galaxy Interactions at Low and High Redshift, Proc. IAU Symposium, No.186, p. 81

Yun, M. S., Ho, P. T. P., \& Lo, K. Y. 1994, Nature, 372, 530 